\title{
Positive solutions for an infinite system of fractional order boundary value problems
}

Fuli Wang ${ }^{1}$ and Yujun Cui ${ }^{2 *}$ (B)

\section{*Correspondence:}

cyj720201@163.com

${ }^{2}$ State Key Laboratory of Mining Disaster Prevention and Control Co-founded by Shandong Province and the Ministry of Science and Technology, Shandong University of Science and Technology, Qingdao, P.R. China

Full list of author information is available at the end of the article

\section{空 Springer}

\begin{abstract}
In this paper, we investigate the existence of positive solutions for an infinite system of fractional order boundary value problems in a Banach sequence space c. Our analysis relies on the Krasnosel'skii fixed point theorem in a cone in conjunction with the criterion of relative compactness in $C^{1}(J, \mathbf{c})$. Finally, an example is given to illustrate our abstract results.
\end{abstract}

MSC: Primary 34A34; secondary 34B18; 46B45

Keywords: Infinite system; Banach sequence spaces; Positive solution; Fractional order boundary value problems

\section{Introduction}

In this paper, we investigate the existence of positive solutions for the following infinite system of fractional order boundary value problems:

$$
\left\{\begin{array}{l}
{ }^{c} \mathrm{D}^{\alpha} u_{n}(t)+f_{n}\left(t, u_{1}(t), u_{2}(t), \ldots, u_{1}^{\prime}(t), u_{2}^{\prime}(t), \ldots\right)=0, \quad 0 \leq t \leq 1, \\
u_{n}(0)=a_{n}, \quad u_{n}(1)=b_{n}, \quad n=1,2, \ldots,
\end{array}\right.
$$

where ${ }^{c} \mathrm{D}^{\alpha}$ denotes the Caputo fractional derivative of order $1<\alpha \leq 2$, and $f_{n}:[0,1] \times$ $\mathbb{R}_{+}^{\infty} \times \mathbb{R}^{\infty} \rightarrow \mathbb{R}_{+}$are given functions (for $n=1,2, \ldots$ ) where $\mathbb{R}^{\infty}$ denotes the vector space consisting of all sequences of real numbers and $\mathbb{R}_{+}^{\infty}$ denotes the positive cone of $\mathbb{R}^{\infty}$. For simplicity, we will write $u(t)$ instead of the sequence of real functions $\left(u_{n}(t)\right)_{n \in \mathbb{N}}$, and $u^{\prime}(t)$ will denote the sequence of real functions $\left(u_{n}^{\prime}(t)\right)_{n \in \mathbb{N}}$. Functions such as $f_{n}\left(t, u_{1}(t), u_{2}(t), \ldots, u_{1}^{\prime}(t), u_{2}^{\prime}(t), \ldots\right)$ will be written as $f_{n}\left(t, u(t), u^{\prime}(t)\right)$ in the sequel. By a solution of the infinite system (1.1) we mean that there is a sequence of functions $u(t)=\left(u_{n}(t)\right)$ satisfying (1.1) on the interval $J:=[0,1]$.

The theory of infinite systems of differential equations is an important branch of nonlinear analysis. Some infinite systems of differential equations or integral equations have been investigated in a few of Banach sequence spaces by $[2,4-7,16,17,20]$ etc. On the one hand, there are a number of problems of infinite systems of differential equations arising naturally in the description of physical phenomena, where only positive solutions are meaningful. The research on the boundary value problems of fractional differential equations has received a great attention in the last decade, and a variety of results can be found in $[1,3,8,10,14,18,19,21,22]$ and the references therein. In this paper, motivated by the

(c) The Author(s) 2019. This article is distributed under the terms of the Creative Commons Attribution 4.0 International License (http://creativecommons.org/licenses/by/4.0/), which permits unrestricted use, distribution, and reproduction in any medium, provided you give appropriate credit to the original author(s) and the source, provide a link to the Creative Commons license, and indicate if changes were made. 
above mentioned literature, we investigate the existence of positive solutions to the infinite system (1.1). Our considerations will be placed in the Banach space c consisting of all convergent sequences of real numbers. Our approach mainly depends on the Krasnosel'skii fixed point theorem in a cone in conjunction with the criterion of relative compactness in the Banach space $C^{1}(J, \mathbf{c})$.

\section{Preliminaries}

Let $\mathrm{AC}(J)$ denote the space of real functions which are absolutely continuous on the interval $J$. It is known that $\mathrm{AC}(J)$ coincides with the space of primitives of Lebesgue summable functions (cf. [12, p. 338]). Further, we denote by $\operatorname{AC}^{1}(J)$ the space of real functions $x(t)$ which have continuous derivatives on $J$ such that $x^{\prime}(t) \in \mathrm{AC}(J)$. Moreover, we will denote by $L^{\infty}(J)$ the Banach space consisting of all essentially bounded functions on $J$ and by $L^{1}(J)$ the Banach space consisting of Lebesgue integrable functions on $J$.

Definition 2.1 (Cf. [11, p. 69]) The fractional integral of $\alpha>0$ order of a function $\varphi: J \rightarrow$ $\mathbb{R}$ is given by

$$
\mathbf{I}^{\alpha} \varphi(t):=\frac{1}{\Gamma(\alpha)} \int_{0}^{t}(t-s)^{\alpha-1} \varphi(s) d s,
$$

provided the right-hand side is pointwise defined on $J$, where $\Gamma$ is the gamma function.

Remark 2.1 (Cf. [9, p. 13]) Let $\varphi \in L^{1}(J)$ and $\alpha>0$. Then the integral $\mathbf{I}^{\alpha} \varphi(t)$ exists for almost every $t \in J$. Moreover, the function $\mathbf{I}^{\alpha} \varphi$ itself is also an element of the space $L^{1}(J)$.

Definition 2.2 (Cf. [11, pp. 91-92]) If $\varphi \in \mathrm{AC}^{m-1}(J)$, then the Caputo fractional derivative ${ }^{c} \mathrm{D}^{\alpha} \varphi$ exists almost everywhere on $J$. If $\alpha>0$ is not an integer, then it is expressed as follows:

$$
\left({ }^{c} \mathrm{D}^{\alpha} \varphi\right)(t):=\frac{1}{\Gamma(m-\alpha)} \int_{0}^{t}(t-s)^{m-\alpha-1} \varphi^{(m)}(s) d s,
$$

where $m=[\alpha]+1$ and $[\alpha]$ stands for the largest integer not greater than $\alpha$.

Example 2.1 (Cf. [9, p. 193]) Let $1<\alpha \leq 2$. From the definition of the Caputo fractional derivative, we quote for $\varphi(t)=t^{\delta}$, then

$$
{ }^{c} \mathrm{D}^{\alpha}\left(t^{\delta}\right)= \begin{cases}0, & \delta=0,1 ; \\ \frac{\Gamma(\delta+1)}{\Gamma(\delta+1-\alpha)} t^{\delta-\alpha}, & \delta>1 .\end{cases}
$$

Lemma 2.1 (Cf. [11, pp. 95-96]) Let $1<\alpha \leq 2$. The following relations hold:

(i) if $y \in L^{\infty}(J)$, then ${ }^{c} \mathrm{D}^{\alpha} \mathbf{I}^{\alpha} y(t)=y(t)$;

(ii) if $y \in \mathrm{AC}^{1}(J)$, then $\mathbf{I}^{\alpha c} \mathrm{D}^{\alpha} y(t)=y(t)-y(0)-y^{\prime}(0) t$.

Lemma 2.2 Let $1<\alpha \leq 2$. If $\psi \in L^{\infty}(J)$, then $y \in \mathrm{AC}^{1}(J)$ is a solution of the boundary value problem

$$
\left\{\begin{array}{l}
{ }^{c} \mathrm{D}^{\alpha} y(t)+\psi(t)=0, \quad t \in J, \\
y(0)=y_{0}, \quad y(1)=y_{1},
\end{array}\right.
$$


if and only if y satisfies

$$
y(t)=\int_{0}^{1} G(t, s) \psi(s) d s+\left(y_{1}-y_{0}\right) t+y_{0}, \quad t \in J,
$$

where the Green's function associated with (2.1) is defined by

$$
G(t, s)=\frac{1}{\Gamma(\alpha)} \begin{cases}t(1-s)^{\alpha-1}-(t-s)^{\alpha-1}, & 0 \leq s \leq t \leq 1, \\ t(1-s)^{\alpha-1}, & 0 \leq t \leq s \leq 1 .\end{cases}
$$

Proof By Lemma 2.1(ii), we deduce from (2.1) that

$$
0=\mathbf{I}^{\alpha c} \mathrm{D}^{\alpha} y(t)+\mathbf{I}^{\alpha} \psi(t)=y(t)-y^{\prime}(0) t-y(0)+\int_{0}^{t} \frac{(t-s)^{\alpha-1}}{\Gamma(\alpha)} \psi(s) d s,
$$

and the boundary conditions give

$$
y(0)=y_{0}, \quad y^{\prime}(0)=y_{1}-y_{0}+\int_{0}^{1} \frac{(1-s)^{\alpha-1}}{\Gamma(\alpha)} \psi(s) d s .
$$

Thus, we obtain that

$$
\begin{aligned}
y(t) & =-\int_{0}^{t} \frac{(t-s)^{\alpha-1}}{\Gamma(\alpha)} \psi(s) d s+t \int_{0}^{1} \frac{(1-s)^{\alpha-1}}{\Gamma(\alpha)} \psi(s) d s+\left(y_{1}-y_{0}\right) t+y_{0} \\
& =\int_{0}^{1} G(t, s) \psi(s) d s+\left(y_{1}-y_{0}\right) t+y_{0}, \quad t \in J .
\end{aligned}
$$

Inversely, if $y \in \mathrm{AC}^{1}(J)$ satisfies (2.2), then we have

$$
y(t)=-\mathbf{I}^{\alpha} \psi(t)+\left(\int_{0}^{1} \frac{(1-s)^{\alpha-1}}{\Gamma(\alpha)} \psi(s) d s+y_{1}-y_{0}\right) t+y_{0} .
$$

The boundary conditions are easy to be verified. Further, by Lemma 2.1(i) and Example 2.1 (which shows that ${ }^{c} \mathrm{D}^{\alpha} t={ }^{c} \mathrm{D}^{\alpha} 1=0$ ), we have

$$
{ }^{c} \mathrm{D}^{\alpha} y(t)=-\psi(t), \quad t \in J,
$$

that is, $y$ is a solution of $(2.1)$.

Remark 2.2 Clearly, the Green's function $G(t, s)$ defined by (2.3) is nonnegative and continuous on $J \times J$.

A simple calculation yields

$$
\frac{\partial G(t, s)}{\partial t}=\frac{1}{\Gamma(\alpha)} \begin{cases}(1-s)^{\alpha-1}-(\alpha-1)(t-s)^{\alpha-2}, & 0 \leq s<t \leq 1 \\ (1-s)^{\alpha-1}, & 0 \leq t \leq s \leq 1 .\end{cases}
$$

The following estimates hold:

$$
\int_{0}^{1} G(t, s) d s \leq \frac{\alpha-1}{\Gamma(\alpha+1)}, \quad \int_{0}^{1}\left|\frac{\partial G(t, s)}{\partial t}\right| d s \leq \frac{\alpha+1}{\Gamma(\alpha+1)}, \quad \forall t \in J .
$$


Further, for $\forall t_{1}, t_{2} \in J$, we have the estimates:

$$
\begin{aligned}
& \int_{0}^{1}\left|G\left(t_{1}, s\right)-G\left(t_{2}, s\right)\right| d s \leq \frac{\left|t_{1}-t_{2}\right|+\left|t_{1}^{\alpha}-t_{2}^{\alpha}\right|}{\Gamma(\alpha+1)}, \\
& \int_{0}^{1}\left|\frac{\partial G\left(t_{1}, s\right)}{\partial t}-\frac{\partial G\left(t_{2}, s\right)}{\partial t}\right| d s \leq \frac{\left|t_{1}^{\alpha-1}-t_{2}^{\alpha-1}\right|}{\Gamma(\alpha)} .
\end{aligned}
$$

Let $\left(E,\|\cdot\|_{E}\right)$ be an infinite dimensional real Banach space. We denote by $C(J, E)$ the space consisting of all functions defined and continuous on the interval $J$ with values in the space $E$. Further, we denote by $C^{1}(J, E)$ the space consisting of all functions defined and continuous differentiable on $J$ with values in the space $E$. It is well known that $C^{1}(J, E)$ is a Banach space equipped with the standard norm

$$
\|u\|_{C^{1}}=\|u\|_{C}+\left\|u^{\prime}\right\|_{C^{\prime}}
$$

where $\|v\|_{C}=\sup _{t \in J}\|v(t)\|_{E}$ for $v \in C(J, E)$.

For a set $\Omega$ in $C^{1}(J, E)$, we will denote by $\Omega^{\prime}$ the set situated in $C(J, E)$ and by $\Omega(t)$ the set situated in $E$ for fixed $t \in J$ as the following forms:

$$
\Omega^{\prime}=\left\{u^{\prime}: u \in \Omega\right\}, \quad \Omega(t)=\{u(t): u \in \Omega\} .
$$

In what follows, we will use the following criterion of compactness in $C^{1}(J, E)$ which is a generalization of the well-known Arzelà-Ascoli theorem.

Theorem 2.1 (Cf. [15, Proposition 1.7]) A bounded subset $\Omega$ of the space $C^{1}(J, E)$ is relatively compact if and only if all functions from $\Omega$ and $\Omega^{\prime}$ are equicontinuous on $J$, and the set $\Omega(t)$ is relatively compact in the space $E$ for each $t \in J$.

In the sequel, we will denote by $\left(\mathbf{c},\|\cdot\|_{\infty}\right)$ the Banach space consisting of all convergent sequences $\xi=\left(\xi_{n}\right)$ with the norm $\|\xi\|_{\infty}=\sup \left\{\left|\xi_{1}\right|,\left|\xi_{2}\right|, \ldots\right\}$.

Let us recall [4, Theorem 3.1] that a bounded subset $X$ of the space $\left(\mathbf{c},\|\cdot\|_{\infty}\right)$ is relatively compact if and only if

$$
\lim _{k \rightarrow \infty}\left[\sup _{\left(\xi_{n}\right) \in X}\left\{\sup \left\{\left|\xi_{m}-\xi_{n}\right|: m, n \geq k\right\}\right\}\right]=0
$$

Further, we will denote by $C^{1}(J, \mathbf{c})$ the Banach space consisting of all functions defined and continuous differentiable on $J$ with values of each function and its derivative in the space $\left(\mathbf{c},\|\cdot\|_{\infty}\right)$, equipped with the norm

$$
\|u\|_{*}:=\|u\|_{C}+\left\|u^{\prime}\right\|_{C}, \quad u \in C^{1}(J, \mathbf{c})
$$

where $\|u\|_{C}=\max _{t \in J}\|u(t)\|_{\infty}$ and $\left\|u^{\prime}\right\|_{C}=\max _{t \in J}\left\|u^{\prime}(t)\right\|_{\infty}$.

The following Krasnosel'skii fixed point theorem in a cone (cf. [13]) will be utilized in our considerations.

Theorem 2.2 Let $\mathcal{E}$ be a Banach space, and let $\mathcal{P} \subseteq \mathcal{E}$ be a cone in $\mathcal{E}$. Assume that $\Omega_{1}$, $\Omega_{2}$ are open subsets of $\mathcal{E}$ with $0 \in \Omega_{1} \subseteq \bar{\Omega}_{1} \subseteq \Omega_{2}$, and let $F: \mathcal{P} \rightarrow \mathcal{P}$ be a completely continuous operator such that, either 
(i) $\|F x\| \leq\|x\|, x \in \mathcal{P} \cap \partial \Omega_{1}$ and $\|F x\| \geq\|x\|, x \in \mathcal{P} \cap \partial \Omega_{2}$, or

(ii) $\|F x\| \geq\|x\|, x \in \mathcal{P} \cap \partial \Omega_{1}$ and $\|F x\| \leq\|x\|, x \in \mathcal{P} \cap \partial \Omega_{2}$.

Then $F$ has a fixed point in $\mathcal{P} \cap\left(\bar{\Omega}_{2} \backslash \Omega_{1}\right)$.

\section{Main results}

Solutions to the infinite system (1.1) will be sought in the cone $\mathcal{P}$ of the Banach space $C^{1}(J, \mathbf{c})$, where $\mathcal{P}$ is defined by

$$
\mathcal{P}:=\left\{u=\left(u_{n}\right) \in C^{1}(J, \mathbf{c}): u_{n} \in \mathrm{AC}^{1}(J), u_{n}(t) \geq 0, t \in J, n=1,2, \ldots\right\} .
$$

In order to investigate the existence of positive solutions to (1.1) in the cone $\mathcal{P}$, we will impose the following hypotheses.

(H1) For each $n \in \mathbb{N}$, the function $f_{n}: J \times \mathbb{R}_{+}^{\infty} \times \mathbb{R}^{\infty} \rightarrow \mathbb{R}_{+}$is such that $f_{n}(\cdot, u, v)$ is measurable for every $(u, v) \in \mathbb{R}_{+}^{\infty} \times \mathbb{R}^{\infty}$, and the sequences of nonnegative real numbers $a=\left(a_{n}\right)$ and $b=\left(b_{n}\right)$ are convergent, and $a \neq \mathbf{0}$ or $b \neq \mathbf{0}$ (where $\mathbf{0}$ denotes the zero sequence).

(H2) The operator $f$ defined on the space $J \times \mathbf{c} \times \mathbf{c}$ as

$$
f(u, v)(t):=\left(f_{1}(t, u, v), f_{2}(t, u, v), \ldots\right)
$$

is such that the class of all functions $\{f(u, v)(t)\}_{t \in J}$ is equicontinuous at every point of the space $\mathbf{c} \times \mathbf{c}$.

(H3) There exist two sequences of continuous functions $\left(p_{n}\right)$ and $\left(q_{n}\right)$, which are convergent uniformly on $J$, such that

$$
\left|f_{n}(t, u, v)\right| \leq\left|p_{n}(t)\right|+\left|q_{n}(t)\right|\left(\|u\|_{\infty}+\|v\|_{\infty}\right)
$$

for $\forall(t, u, v) \in J \times \mathbf{c} \times \mathbf{c}$.

Moreover, for any bounded subset $X$ of the space $\mathbf{c}$, we assume that the sequence $\left(f_{n}(t, u, v)\right)$ is convergent uniformly on $J \times X \times X$, i.e., for arbitrary given $\varepsilon>0$, there exists $n_{0} \in \mathbb{N}$ such that

$$
\left|f_{m}(t, u, v)-f_{n}(t, u, v)\right| \leq \varepsilon, \quad \text { as } m, n \geq n_{0},
$$

for $\forall(t, u, v) \in J \times X \times X$.

Let us introduce the following notations:

$$
\begin{array}{ll}
p(t)=\left(p_{n}(t)\right), \quad P=\sup _{t \in J} \int_{0}^{1}\left(G(t, s)+\left|\frac{\partial G(t, s)}{\partial t}\right|\right)\|p(s)\|_{\infty} d s \\
q(t)=\left(q_{n}(t)\right), \quad Q=\sup _{t \in J} \int_{0}^{1}\left(G(t, s)+\left|\frac{\partial G(t, s)}{\partial t}\right|\right)\|q(s)\|_{\infty} d s .
\end{array}
$$

Now, we define the operator $\mathcal{F}$ on $C^{1}(J, \mathbf{c})$ by $\mathcal{F} u:=\left(F_{1} u, F_{2} u, \ldots\right)$ such that, for each $n \in \mathbb{N}$, the function $F_{n}: C^{1}(J, \mathbf{c}) \rightarrow \mathbb{R}$ is expressed in the form

$$
F_{n} u(t)=\int_{0}^{1} G(t, s) f_{n}\left(s, u(s), u^{\prime}(s)\right) d s+b_{n} t+a_{n}(1-t),
$$


where the Green's function is defined by (2.3). Then we have

$$
\left(F_{n} u\right)^{\prime}(t)=\int_{0}^{1} \frac{\partial G(t, s)}{\partial t} f_{n}\left(s, u(s), u^{\prime}(s)\right) d s+b_{n}-a_{n}, \quad n=1,2, \ldots
$$

By Lemma 2.2, it is clear that there exist positive solutions to (1.1) if and only if the operator $\mathcal{F}$ has fixed points in the cone $\mathcal{P}$, that is, there exists $u \in \mathcal{P}(u \neq \mathbf{0})$ such that $F_{n} u=u_{n}(n=1,2, \ldots)$.

Lemma 3.1 Under assumptions $(\mathbf{H} 1)-(\mathbf{H} 3)$, the operator $\mathcal{F}: \mathcal{P} \rightarrow \mathcal{P}$ defined by (3.3) is completely continuous in the Banach space $C^{1}(\mathrm{~J}, \mathbf{c})$.

Proof Let $u \in \mathcal{P}$ be arbitrarily fixed. In view of expressions (3.3) and (3.4), it may infer that $F_{n} u \in \mathrm{AC}^{1}(J)$ and $F_{n} u(t) \geq 0(n=1,2, \ldots)$ on account of our assumptions.

According to the equicontinuity of the operator $f$ at point $\left(u, u^{\prime}\right)$ by $(\mathbf{H} 2)$, for given $\varepsilon>0$, there exists $\delta>0$ such that

$$
\left\|f\left(u, u^{\prime}\right)(s)-f\left(v, v^{\prime}\right)(s)\right\|_{\infty} \leq \frac{\Gamma(\alpha)}{2} \varepsilon
$$

whenever $\|u-v\|_{C} \leq \delta$ and $\left\|u^{\prime}-v^{\prime}\right\|_{C} \leq \delta$ for $v \in \mathcal{P}$ and $\forall s \in J$. Then we deduce from (2.4) that

$$
\begin{aligned}
\|\mathcal{F} u-\mathcal{F} v\|_{*} \leq & \sup _{t \in J} \int_{0}^{1} G(t, s)\left\|f\left(u, u^{\prime}\right)(s)-f\left(v, v^{\prime}\right)(s)\right\|_{\infty} d s \\
& +\sup _{t \in J} \int_{0}^{1}\left|\frac{\partial G(t, s)}{\partial t}\right|\left\|f\left(u, u^{\prime}\right)(s)-f\left(v, v^{\prime}\right)(s)\right\|_{\infty} d s \\
\leq & \left(\sup _{t \in J} \int_{0}^{1} G(t, s) d s+\sup _{t \in J} \int_{0}^{1}\left|\frac{\partial G(t, s)}{\partial t}\right| d s\right) \frac{\Gamma(\alpha)}{2} \varepsilon \leq \varepsilon,
\end{aligned}
$$

which implies that $\mathcal{F}: \mathcal{P} \rightarrow \mathcal{P}$ is continuous.

Next, let $\Omega \subseteq \mathcal{P}$ be a bounded set. It follows that there exists $M>0$ such that $\|u\|_{*} \leq M$ for $\forall u \in \Omega$. Then, for given $u \in \Omega$ and $n \in \mathbb{N}$, we deduce from (3.1) that

$$
\begin{aligned}
\left|F_{n} u(t)\right| \leq & \int_{0}^{1} G(t, s) f_{n}\left(s, u(s), u^{\prime}(s)\right) d s+b_{n} t+a_{n}(1-t) \\
\leq & \int_{0}^{1} G(t, s)\left[p_{n}(s)+q_{n}(s)\left(\|u(s)\|_{\infty}+\left\|u^{\prime}(s)\right\|_{\infty}\right)\right] d s \\
& +b_{n} t+a_{n}(1-t) \\
\leq & \int_{0}^{1} G(t, s)\|p(s)\|_{\infty} d s+M \int_{0}^{1} G(t, s)\|q(s)\|_{\infty} d s \\
& +\|b\|_{\infty} t+\|a\|_{\infty}(1-t) .
\end{aligned}
$$

Similarly, we have

$$
\begin{aligned}
\left|\left(F_{n} u\right)^{\prime}(t)\right| \leq & \int_{0}^{1}\left|\frac{\partial G(t, s)}{\partial t}\right|\|p(s)\|_{\infty} d s+M \int_{0}^{1}\left|\frac{\partial G(t, s)}{\partial t}\right|\|q(s)\|_{\infty} d s \\
& +\|b\|_{\infty}+\|a\|_{\infty} .
\end{aligned}
$$


Thus, we obtain that

$$
\|\mathcal{F} u\|_{*} \leq P+Q M+2\|b\|_{\infty}+2\|a\|_{\infty}, \quad \forall u \in \Omega,
$$

which implies that $\mathcal{F}$ maps bounded sets of the cone $\mathcal{P}$ into bounded ones.

Let $\forall t_{1}, t_{2} \in J$. For an arbitrary fixed $u \in \Omega$ we deduce from (2.5) that

$$
\begin{aligned}
& \left|F_{n} u\left(t_{1}\right)-F_{n} u\left(t_{2}\right)\right| \\
& \quad \leq \int_{0}^{1}\left|G\left(t_{1}, s\right)-G\left(t_{2}, s\right)\right| f_{n}\left(s, u(s), u^{\prime}(s)\right) d s+\left|b_{n}-a_{n}\right| \cdot\left|t_{1}-t_{2}\right| \\
& \quad \leq\left(\frac{\|p\|_{C}+M\|q\|_{C}}{\Gamma(\alpha+1)}+\|b-a\|_{\infty}\right)\left(\left|t_{1}-t_{2}\right|+\left|t_{1}^{\alpha}-t_{2}^{\alpha}\right|\right), \quad n=1,2, \ldots ;
\end{aligned}
$$

and

$$
\begin{aligned}
\left|\left(F_{n} u\right)^{\prime}\left(t_{1}\right)-\left(F_{n} u\right)^{\prime}\left(t_{2}\right)\right| & \leq \int_{0}^{1}\left|\frac{\partial G\left(t_{1}, s\right)}{\partial t}-\frac{\partial G\left(t_{2}, s\right)}{\partial t}\right| f_{n}\left(s, u(s), u^{\prime}(s)\right) d s \\
& \leq \frac{\|p\|_{C}+M\|q\|_{C}}{\Gamma(\alpha)}\left|t_{1}^{\alpha-1}-t_{2}^{\alpha-1}\right|, \quad n=1,2, \ldots
\end{aligned}
$$

which imply that all functions from the sets $\mathcal{F} \Omega$ and $(\mathcal{F} \Omega)^{\prime}$ are equicontinuous on the interval $J$.

Now, for fixed $t \in J$, let us consider the set $\mathcal{F} \Omega(t)$. For $\forall u \in \Omega$, we have

$$
\begin{aligned}
\left|F_{m} u(t)-F_{n} u(t)\right| \leq & \int_{0}^{1} G(t, s)\left|f_{m}\left(s, u(s), u^{\prime}(s)\right)-f_{n}\left(s, u(s), u^{\prime}(s)\right)\right| d s \\
& +\left|b_{m}-b_{n}\right| t+\left|a_{m}-a_{n}\right|(1-t), \quad \forall m, n \in \mathbb{N} .
\end{aligned}
$$

From estimate (3.2) and the convergence of $\left(a_{n}\right)$ and $\left(b_{n}\right)$, we conclude that

$$
\lim _{k \rightarrow \infty}\left[\sup _{u \in \Omega}\left\{\sup \left\{\left|F_{m} u(t)-F_{n} u(t)\right|: m, n \geq k\right\}\right\}\right]=0
$$

which shows that $\mathcal{F} \Omega(t)$ is relatively compact in the space $\left(\mathbf{c},\|\cdot\|_{\infty}\right)$ for each $t \in J$ by formula (2.6).

Taking into account the fact that the sets $\mathcal{F} \Omega$ and $(\mathcal{F} \Omega)^{\prime}$ consist of functions equicontinuous on the interval $J$, on the base of Theorem 2.1, we infer that the set $\mathcal{F} \Omega$ is relatively compact in the Banach space $C^{1}(J, \mathbf{c})$. Consequently, we show that $\mathcal{F}: \mathcal{P} \rightarrow \mathcal{P}$ is completely continuous.

Now we are in a position to state our main result.

Theorem 3.1 Under assumptions $(\mathbf{H} 1)-(\mathbf{H} 3)$, if additionally $Q<1$, the infinite system (1.1) has at least one positive solution $u(t)=\left(u_{n}(t)\right)$, and $\left(u_{n}(t)\right)$ is a convergent sequence, i.e., $\left(u_{n}(t)\right) \in \mathbf{c}$ for each $t \in J$. 
Proof Let $r_{1}:=\max \left\{\|a\|_{\infty},\|b\|_{\infty}\right\}$ and $\Omega_{1}:=\left\{u \in C^{1}(J, \mathbf{c}):\|u\|_{*}<r_{1}\right\}$. For $u \in \mathcal{P} \cap \partial \Omega_{1}$, we have $\|u\|_{*}=r_{1}$. According to the nonnegativity of $f_{n}$ and $G(t, s)$, we deduce from (3.3) that

$$
F_{n} u(t) \geq b_{n} t+a_{n}(1-t), \quad n=1,2, \ldots,
$$

it follows that

$$
\|\mathcal{F} u\|_{*} \geq \sup _{t \in J}\left\{\|b\|_{\infty} t+\|a\|_{\infty}(1-t)\right\}=\max \left\{\|a\|_{\infty},\|b\|_{\infty}\right\}=r_{1}=\|u\|_{*} .
$$

Let

$$
r_{2}:=\frac{P+2\|b\|_{\infty}+2\|a\|_{\infty}}{1-Q},
$$

and let $\Omega_{2}:=\left\{u \in C^{1}(J, \mathbf{c}):\|u\|_{*}<r_{2}\right\}$. For $u \in \mathcal{P} \cap \partial \Omega_{2}$, we have $\|u\|_{*}=r_{2}$. Similar to estimate (3.5) we have

$$
\|\mathcal{F} u\|_{*} \leq P+Q r_{2}+2\|b\|_{\infty}+2\|a\|_{\infty}=r_{2}=\|u\|_{*} .
$$

Since $\bar{\Omega}_{1} \subset \Omega_{2}$, then by the second part of Theorem 2.1, it follows that $\mathcal{F}$ has a fixed point $u$ in $\mathcal{P} \cap\left(\bar{\Omega}_{2} \backslash \Omega_{1}\right)$. Thus, $u(t)=\left(u_{n}(t)\right)$ is a positive solution of the infinite system (1.1), and $u(t) \in \mathbf{c}$ for each $t \in J$.

Example 3.1 Consider the following infinite system of fractional order boundary value problems:

$$
\begin{aligned}
& { }^{c} \mathrm{D}^{3 / 2} u_{n}(t)+\sum_{k=0}^{n} \frac{t^{k}}{k !}+\frac{t^{n+1}}{2^{n+1}} \frac{\left|u_{n}^{\prime}(t)\right|}{1+\left|u_{n}(t)\right|}+\sum_{k=n+1}^{\infty} \frac{t^{k+1}}{2^{k+1}} \frac{u_{k}(t)}{1+\left|u_{k}(t)\right|}=0, \\
& u_{n}(0)=\frac{1}{n}, \quad u_{n}(1)=\frac{n}{n+1}, \quad 0 \leq t \leq 1, n=1,2, \ldots
\end{aligned}
$$

In order to show that this system admits a positive solution in $C^{1}(J, \mathbf{c})$, we are going to check the conditions of Theorem 3.1. To this end, we define the functions and sequences as follows:

$$
\begin{aligned}
& f_{n}(t, u, v):=\sum_{k=0}^{n} \frac{t^{k}}{k !}+\frac{t^{n+1}}{2^{n+1}} \frac{\left|v_{n}\right|}{1+\left|u_{n}\right|}+\sum_{k=n+1}^{\infty} \frac{t^{k+1}}{2^{k+1}} \frac{u_{k}}{1+\left|u_{k}\right|}, \\
& a_{n}:=\frac{1}{n}, \quad b_{n}:=\frac{n}{n+1}, \quad n=1,2, \ldots
\end{aligned}
$$

Clearly, $f_{n}: J \times \mathbb{R}_{+}^{\infty} \times \mathbb{R}^{\infty} \rightarrow \mathbb{R}_{+}$is measurable for each $n \in \mathbb{N}$, and the sequences $a=\left(a_{n}\right)$, $b=\left(b_{n}\right)$ are convergent. Thus, assumption $(\mathbf{H} 1)$ is verified.

For a fixed point $(\widetilde{u}, \widetilde{v}) \in \mathbf{c} \times \mathbf{c}$ and given $\varepsilon>0$, choose $\delta=\varepsilon / 2\left(1+\|v\|_{\infty}\right)$ and take $(u, v) \in$ $\mathbf{c} \times \mathbf{c}$ such that $\|u-\widetilde{u}\|_{\infty} \leq \delta$ and $\|v-\widetilde{v}\|_{\infty} \leq \delta$. Then, for each $n \in \mathbb{N}$, we have the following estimate:

$$
\begin{aligned}
& \left|f_{n}(t, u, v)-f_{n}(t, \widetilde{u}, \widetilde{v})\right| \\
& \quad \leq \frac{t^{n+1}}{2^{n+1}}\left(\frac{\left|v_{n}-\widetilde{v}_{n}\right|}{1+\left|u_{n}\right|}+\frac{\left|\widetilde{v}_{n}\right|\left|u_{n}-\widetilde{u}_{n}\right|}{\left(1+\left|u_{n}\right|\right)\left(1+\left|\widetilde{u}_{n}\right|\right)}\right)
\end{aligned}
$$




$$
\begin{aligned}
& +\sum_{k=n+1}^{\infty} \frac{t^{k+1}}{2^{k+1}}\left(\frac{\left|u_{k}-\widetilde{u}_{k}\right|}{1+\left|u_{k}\right|}+\frac{\left|\widetilde{u}_{k}\right|\left|u_{k}-\widetilde{u}_{k}\right|}{\left(1+\left|u_{k}\right|\right)\left(1+\left|\widetilde{u}_{k}\right|\right)}\right) \\
\leq & \frac{t^{n+1}}{2^{n}}\left(\|v-\widetilde{v}\|_{\infty}+\|\widetilde{v}\|_{\infty}\|u-\widetilde{u}\|_{\infty}\right)+\left(\sum_{k=n+1}^{\infty} \frac{t^{k+1}}{2^{k}}\right)\|u-\widetilde{u}\|_{\infty} \\
\leq & \left(\sum_{k=1}^{\infty} \frac{t^{k+1}}{2^{k}}\right)\left[\left(1+\|v\|_{\infty}\right)\|u-\widetilde{u}\|_{\infty}+\|v-\widetilde{v}\|_{\infty}\right] \leq \varepsilon,
\end{aligned}
$$

which implies that the class of functions $\{f(u, v)(t)\}_{t \in J}$ is equicontinuous at $(\widetilde{u}, \widetilde{v})$. Thus, assumption (H2) is verified.

Next, let us define the sequences of functions $\left(p_{n}\right)$ and $\left(q_{n}\right)$ as follows:

$$
p_{n}(t):=\sum_{k=0}^{n} \frac{t^{k}}{k !}, \quad q_{n}(t):=\sum_{k=n}^{\infty} \frac{t^{k+1}}{2^{k}}, \quad n=1,2, \ldots
$$

Let $X$ be a bounded subset of the space c. We assume $\|u\|_{\infty} \leq M$ for $\forall u \in X$. Thus, for $\forall m, n \in \mathbb{N}$ with $m>n$ and $u, v \in X$, we get

$$
\begin{aligned}
& \left|f_{m}(t, u, v)-f_{n}(t, u, v)\right| \\
& \quad \leq \sum_{k=n+1}^{m} \frac{t^{k}}{k !}+\frac{t^{n+1}}{2^{n+1}}\left(\frac{\left|v_{m}\right|}{1+\left|u_{m}\right|}+\frac{\left|v_{n}\right|}{1+\left|u_{n}\right|}\right)+\sum_{k=n+1}^{m} \frac{t^{k+1}}{2^{k+1}} \cdot \frac{\left|u_{k}\right|}{1+\left|u_{k}\right|} \\
& \quad \leq \sum_{k=n+1}^{m} \frac{t^{k}}{k !}+\left(\sum_{k=n}^{m} \frac{t^{k+1}}{2^{k}}\right)\left(\|u\|_{\infty}+\|v\|_{\infty}\right) \\
& \quad \leq \sum_{k=n+1}^{m} \frac{t^{k}}{k !}+2 M\left(\sum_{k=n}^{m} \frac{t^{k+1}}{2^{k}}\right),
\end{aligned}
$$

then the uniform convergence of $\left(p_{n}\right)$ and $\left(q_{n}\right)$ implies that estimate (3.2) of $(\mathbf{H} 3)$ is satisfied for $\forall t \in J$. Further, we conclude that estimate (3.1) of (H3) is satisfied from the following inequality:

$$
\begin{aligned}
\left|f_{n}(t, u, v)\right| & \leq \sum_{k=0}^{n} \frac{t^{k}}{k !}+\frac{t^{n+1}}{2^{n+1}}\|v\|_{\infty}+\left(\sum_{k=n+1}^{\infty} \frac{t^{k+1}}{2^{k+1}}\right)\|u\|_{\infty} \\
& \leq \sum_{k=0}^{n} \frac{t^{k}}{k !}+\left(\sum_{k=n}^{\infty} \frac{t^{k+1}}{2^{k}}\right)\left(\|u\|_{\infty}+\|v\|_{\infty}\right), \quad \forall t \in J .
\end{aligned}
$$

Moreover, from $\|q(t)\|_{\infty}=t^{2} /(2-t) \leq t^{2}$ we deduce that

$$
\begin{aligned}
& \int_{0}^{1}\left(G(t, s)+\left|\frac{\partial G(t, s)}{\partial t}\right|\right) \frac{s^{2}}{2-s} d s \\
& \quad \leq \int_{0}^{1}\left(G(t, s)+\left|\frac{\partial G(t, s)}{\partial t}\right|\right) s^{2} d s \\
& \quad \leq \int_{0}^{1} \frac{t(1-s)^{\frac{1}{2}} s^{2}}{\Gamma(3 / 2)} d s-\int_{0}^{t} \frac{(t-s)^{\frac{1}{2}} s^{2}}{\Gamma(3 / 2)} d s+\int_{0}^{1} \frac{(1-s)^{\frac{1}{2}} s^{2}}{\Gamma(3 / 2)} d s+\int_{0}^{t} \frac{(t-s)^{-\frac{1}{2}} s^{2}}{2 \Gamma(3 / 2)} d s
\end{aligned}
$$




$$
\begin{aligned}
& =\frac{2}{\Gamma(9 / 2)}\left(t-t^{7 / 2}+1+\frac{7}{2} t^{5 / 2}\right) \\
& \leq \frac{9}{\Gamma(9 / 2)}=0.773 \ldots, \quad \forall t \in J
\end{aligned}
$$

\section{it follows that $Q<1$.}

Now, based on Theorem 3.1, we infer that there exists at least one positive solution to the infinite system (3.6), and for each $t \in J$, the solution $\left(u_{n}(t)\right)$ forms a convergent sequence.

\section{Acknowledgements}

The authors wish to thank anonymous referees for their valuable suggestions.

\section{Funding}

This work was partially supported by the Natural Science Foundation of China (11571207), the Shandong Natural Science Foundation (ZR2018MA011), and the Tai'shan Scholar Engineering Construction Fund of Shandong Province of China.

\section{Availability of data and materials}

Not applicable.

\section{Competing interests}

The authors declare that they have no competing interests.

\section{Authors' contributions}

The authors have made the same contribution. All authors read and approved the final manuscript.

\section{Author details}

'School of Mathematics and Physics, Changzhou University, Changzhou, P.R. China. ${ }^{2}$ State Key Laboratory of Mining Disaster Prevention and Control Co-founded by Shandong Province and the Ministry of Science and Technology, Shandong University of Science and Technology, Qingdao, P.R. China.

\section{Publisher's Note}

Springer Nature remains neutral with regard to jurisdictional claims in published maps and institutional affiliations.

Received: 24 January 2019 Accepted: 21 April 2019 Published online: 03 May 2019

\section{References}

1. Agarwal, R.P., Benchohra, M., Hamani, S.: A survey on existence results for boundary value problems of nonlinear fractional differential equations and inclusions. Acta Appl. Math. 109, 973-1033 (2010)

2. Aghajani, A., Pourhadi, E.: Application of measure of noncompactness to $\ell_{1}$-solvability of infinite systems of second order differential equations. Bull. Belg. Math. Soc. Simon Stevin 22(1), 105-118 (2015)

3. Bai, Z:: On positive solutions of a nonlocal fractional boundary value problem. Nonlinear Anal. 72, 916-924 (2010)

4. Banaś, J., Krajewska, M.: Existence of solutions for infinite systems of differential equations in spaces of tempered sequences. Electron. J. Differ. Equ. 2017, 60 (2017)

5. Banaś, J., Lecko, M.: Solvability of infinite systems of differential equations in Banach sequence spaces. J. Comput. Appl. Math. 137, 363-375 (2001)

6. Banaś, J., Lecko, M.: An existence theorem for a class of infinite system of integral equations. Math. Comput. Model. $34,535-539(2001)$

7. Banaś, J., Mursaleen, M., Rizvi, S.M.H.: Existence of solutions to a boundary-value problem for an infinite system of differential equations. Electron. J. Differ. Equ. 2017, 262 (2017)

8. Cui, Y:: Uniqueness of solution for boundary value problems for fractional differential equations. Appl. Math. Lett. 51, 48-54 (2016)

9. Diethem, K.: The Analysis of Fractional Differential Equations. Springer, Heidelberg (2004)

10. Henderson, J., Luca, R.: Boundary Value Problems for Systems of Differential, Difference and Fractional Equations, Positive Solutions. Elsevier, Amsterdam (2016)

11. Kilbas, A.A., Srivastava, H.M., Trujillo, J.J.: Theory and Applications of Fractional Differential Equations. Elsevier, Amsterdam (2006)

12. Kolmogorov, A.N., Fomin, S.V.: Fundamentals of the Theory of Functions and Functional Analysis. Nauka, Moscow (1968)

13. Krasnosel'skii, M.A.: Positive Solutions of Operator Equations. Noordhoff, Groningen (1964)

14. Lakshmikantham, V., Leela, S., Vasundhara, J.: Theory of Fractional Dynamic Systems. Cambridge Academic Publishers, Cambridge (2009)

15. Mönch, H.: Boundary value problems for nonlinear ordinary differential equations of second order in Banach spaces. Nonlinear Anal. 4(5), 985-999 (1980)

16. Mursaleen, M., Shole Haghighi, A.: Solvability of infinite systems of second order differential equations in $c_{0}$ and $\ell_{1}$ by Meir-Keeler condensing operators. Proc. Am. Math. Soc. 144, 4279-4289 (2016)

17. Rzepka, B., Sadarangani, K.: On solutions of an infinite system of singular integral equations. Math. Comput. Model. 45, 1265-1271 (2007) 
18. Sun, Q., Ji, H., Cui, Y.: Positive solutions for boundary value problems of fractional differential equation with integral boundary conditions. J. Funct. Spaces 2018, Article ID 6461930 (2018)

19. Sun, Q., Meng, S., Cui, Y.: Existence results for fractional order differential equation with nonlocal Erdélyi-Kober and generalized Riemann-Liouville type integral boundary conditions at resonance. Adv. Differ. Equ. 2018, 243 (2018)

20. Wang, F., Cui, Y.: Solvability for an infinite system of fractional order boundary value problems. Ann. Funct. Anal. (2019) (in press)

21. Zhang, X., Liu, L., Wu, Y., Zou, Y.: Existence and uniqueness of solutions for systems of fractional differential equations with Riemann-Stieltjes integral boundary condition. Adv. Differ. Equ. 2018, 204 (2018)

22. Zuo, M., Hao, X., Liu, L., Cui, Y.: Existence results for impulsive fractional integro-differential equation of mixed type with constant coefficient and antiperiodic boundary conditions. Bound. Value Probl. 2017, 161 (2017)

Submit your manuscript to a SpringerOpen ${ }^{\odot}$ journal and benefit from:

- Convenient online submission

- Rigorous peer review

- Open access: articles freely available online

- High visibility within the field

- Retaining the copyright to your article

Submit your next manuscript at $\boldsymbol{\Delta}$ springeropen.com 